\title{
Topological Semimetals in the SnTe Material Class: Nodal Lines and Weyl Points
}

\author{
Alexander Lau ${ }^{1}$ and Carmine Ortix ${ }^{2,3}$ \\ ${ }^{1}$ Kavli Institute of Nanoscience, Delft University of Technology, P.O. Box 4056, 2600 GA Delft, Netherlands \\ ${ }^{2}$ Institute for Theoretical Physics, Center for Extreme Matter and Emergent Phenomena, Utrecht University, \\ Princetonplein 5, 3584 CC Utrecht, Netherlands \\ ${ }^{3}$ Dipartimento di Fisica "E. R. Caianiello," Universitá di Salerno, IT-84084 Fisciano, Italy
}

(Received 25 April 2018; revised manuscript received 30 July 2018; published 7 May 2019)

\begin{abstract}
We theoretically show that IV-VI semiconducting compounds with low-temperature rhombohedral crystal structure represent a new potential platform for topological semimetals. By means of minimal $\mathbf{k} \cdot \mathbf{p}$ models, we find that the two-step structural symmetry reduction of the high-temperature rocksalt crystal structure, comprising a rhombohedral distortion along the [111] direction followed by a relative shift of the cation and anion sublattices, gives rise to topologically protected Weyl semimetal and nodal line semimetal phases. We derive general expressions for the nodal features and apply our results to SnTe, showing explicitly how Weyl points and nodal lines emerge in this system. Experimentally, the topological semimetals could potentially be realized in the low-temperature ferroelectric phase of $\mathrm{SnTe}, \mathrm{GeTe}$, and related alloys.
\end{abstract}

DOI: 10.1103/PhysRevLett.122.186801

Introduction.-The recent discovery of topological semimetals [1-8], the most prominent examples of which are Weyl semimetals (WSMs) and Dirac semimetals [9-13], has attracted huge interest in gapless topological phases of matter [14-24]. Generally speaking, topological semimetals are systems where the conduction and the valence bands have robust crossings in the Brillouin zone (BZ). In WSMs, these robust crossings - the so-called Weyl nodes-are isolated, twofold degenerate points and generically require the absence of either time-reversal or inversion symmetry $[13,14]$. Furthermore, Weyl points represent monopoles of the Berry flux and, therefore, carry a topological charge $[10,13]$. The topological nature of Weyl nodes leads, by the bulk-boundary correspondence, to the presence of surface Fermi arcs [25] possibly coexisting with surface Dirac cones [26]. In Dirac semimetals, instead, both the conduction and the valence bands are twofold degenerate and cross at isolated fourfold degenerate points in the BZ. As opposed to Weyl nodes, Dirac points are typically unstable degeneracies and can be regarded as the parent semimetallic state generating a WSM by inversion or timereversal symmetry breaking [27]. Various WSM materials have been predicted theoretically $[4,14,17,25,28-34]$ and realized experimentally [3,5-8,35-38]. These include both binary and ternary compounds [39]. Nevertheless, in view of potential applications, it is important to seek new material platforms and novel mechanisms for the realization of WSMs.

A different class of topological semimetals features conduction and valence bands crossing each other along closed lines in the BZ [40-44]. These nodal line semimetals are midway between semimetals with point nodes and metals with a two-dimensional Fermi surface. One of the typical features of nodal line semimetals is the presence of drumhead surface states bounded by the surface projection of the nodal lines, whose stability is guaranteed by the presence of, for instance, mirror symmetries [42,45,46]. In contrast to WSMs, only a few candidate materials for topological nodal line semimetals have been put forward [43].

In this Letter, we show that both nodal line and WSM phases can potentially appear as a result of a structural distortion in group-IV tellurides with high-temperature rocksalt crystal structure, such as $\mathrm{SnTe}, \mathrm{GeTe}$, and related alloys [47-49]. A crystal symmetry reduction to a rhombohedral phase via an elastic strain along the (111) direction reduces the point-group symmetry of a subset of $L$ points in the BZ [50]. We show that this leads to bulk Dirac points close to these high-symmetry points that evolve either into pairs of Weyl nodes or into mirrorsymmetry protected nodal loops upon breaking inversion symmetry. The latter is naturally realized, for instance, via a relative shift of the anion and cation sublattices during a ferroelectric distortion. Our analysis is based on effective $\mathbf{k} \cdot \mathbf{p}$ models describing the low-energy physics close to the $L$ points of the BZ. In particular, we derive general expressions for Weyl points and nodal lines and apply our general results to a specific model based on SnTe. We show explicitly how Weyl points and nodal lines appear and calculate topological invariants associated with the semimetallic phases.

Dirac points by strain engineering.-IV-VI narrow band gap semiconductors have a high-temperature rocksalt lattice structure with a face-centered-cubic BZ [51]. The 
BZ is bounded by six square faces and eight hexagonal faces. The centers of the latter, commonly denoted by $L$, represent high-symmetry points in the BZ with $D_{3 d}$ pointgroup symmetry [50], which is generated by inversion, a $C_{3}$ axis along $\Gamma L$, and a mirror plane containing $\Gamma$ and two $L$ points, hereafter dubbed as $L$ and $L^{\prime}$, related by a $C_{4}$ rotation [51].

Since the fundamental band gap of group-IV tellurides is located at the four equivalent $L$ points related by the pointgroup symmetries of the lattice [52], the band structure close to the Fermi level can be captured within an effective four-band low-energy $\mathbf{k} \cdot \mathbf{p}$ model [51]. We start out by taking this continuum model and augment it by terms quadratic in the momentum $\mathbf{k}$. Taking into account all symmetry constraints, including time-reversal symmetry (see Refs. [53,54] and Supplemental Material [55]), the model reads

$$
\begin{aligned}
H_{0}(\mathbf{k})= & m \sigma_{z}+\nu\left(k_{1} s_{2}-k_{2} s_{1}\right) \sigma_{x}+\nu_{3} k_{3} \sigma_{y} \\
& +c k_{3}^{2} \sigma_{z}+f\left(k_{1}^{2}+k_{2}^{2}\right) \sigma_{z},
\end{aligned}
$$

where, without loss of generality, we have neglected all terms proportional to the identity, since they correspond either to a rigid shift of all energies or to a balanced change in the curvature of all bands. Therefore, they do not affect the band topology. In the chosen coordinate system, $k_{1}$ is perpendicular to the mirror plane, and $k_{3}$ points along the $C_{3}$ axis going through the $L$ point. The $\sigma_{i}$ are Pauli matrices in orbital space spanned by the $p$ orbitals of the cation $(\mathrm{Pb}$, $\mathrm{Sn}, \mathrm{Ge}$ ) and anion (Te), whereas the $s_{i}$ are Pauli matrices in spin space. Because of the simultaneous presence of inversion and time-reversal symmetry, all states are twofold degenerate.

Contrary to the trivial semiconductors $\mathrm{PbTe}$ and $\mathrm{GeTe}$, for SnTe it is well known that an inverted band gap at the $L$ points gives rise to a crystalline topological-insulating phase protected by mirror symmetry [51]. Now, we show that, independent of the band ordering, a structural distortion to a rhombohedral phase via an elastic strain along the cube diagonal, i.e., the [111] direction, can lead to the emergence of bulk Dirac points, i.e., generic fourfold degenerate band crossing points.

The rhombohedral distortion breaks the $C_{4}$ symmetry of the face-centered-cubic lattice [see Fig. 1(a)]. Consequently, the square faces of the BZ distort into rectangles, and the hexagonal faces are no longer identical as is illustrated in Fig. 1(b). Equivalently, the corresponding elastic strain acts differently on different $L$ points [50]: It does not affect the symmetry of the $L$ point in the [111] direction. However, in the local coordinate system of the point $L^{\prime}$, which was previously related to $L$ by a $C_{4}$ rotation, the strain acts in the $[11 \overline{1}]$ direction. In contrast to $L$, this lowers the symmetry group at $L^{\prime}$ from $D_{3 d}$ to $C_{2 h}$ : The $C_{3}$ symmetry with respect to an axis going through $L^{\prime}$ is explicitly broken, whereas mirror and inversion


FIG. 1. (a) Crystal structure of rhombohedrally distorted SnTe. (b) Rhombohedral Brillouin zone with high-symmetry points $L$ and $L^{\prime}$, a local coordinate system at one of the $L^{\prime}$ points, and the strain direction (red arrow) responsible for the distortion from a face-centered-cubic to a rhombohedral lattice. Also highlighted is one of the three mirror planes spanned by $\Gamma, L$, and $L^{\prime}$.

symmetry are still preserved. Equivalently, the symmetry group at the other two $L^{\prime}$ points is lowered to $C_{2 h}$. As a result, there are now one $L$ point with $D_{3 d}$ symmetry and three $L^{\prime}$ points with $C_{2 h}$ symmetry.

The reduction of point-group symmetry at the $L^{\prime}$ points gives rise to additional symmetry-allowed terms in the corresponding $\mathbf{k} \cdot \mathbf{p}$ theory. Up to second order in $\mathbf{k}$, they read

$$
\begin{aligned}
H_{1}(\mathbf{k})= & \delta \nu\left(k_{1} s_{2}+k_{2} s_{1}\right) \sigma_{x}+\lambda_{1} k_{1} s_{3} \sigma_{x}+\lambda_{2} k_{2} \sigma_{y} \\
& +\lambda_{3} k_{3} s_{1} \sigma_{x}+\delta f\left(k_{1}^{2}-k_{2}^{2}\right) \sigma_{z}+g k_{2} k_{3} \sigma_{z} .
\end{aligned}
$$

We now show that the extended model given by $\tilde{H}=$ $H_{0}+H_{1}$ gives rise to isolated bulk Dirac points. The energies of $\tilde{H}$ can be written as

$$
\begin{aligned}
E^{2}= & \left(f_{1} k_{1}^{2}+f_{2} k_{2}^{2}+g k_{2} k_{3}+c k_{3}^{2}+m\right)^{2}+k_{1}^{2}\left(\lambda_{1}^{2}+\nu_{1}^{2}\right) \\
& +\left(k_{2} \lambda_{2}+k_{3} \nu_{3}\right)^{2}+\left(k_{3} \lambda_{3}-k_{2} \nu_{2}\right)^{2},
\end{aligned}
$$

where we have defined $\nu_{1,2}=\nu \pm \delta \nu$ and $f_{1,2}=f \pm \delta f$. The spectrum is symmetric under $E \rightarrow-E$. Hence, Dirac points, if present, will be located at $E=0$. It is straightforward to see that the spectrum has Dirac points if $E$ is of the form $\pm \sqrt{\left(a k^{2}-m\right)^{2}}$. This implies that all binomials under the square root have to vanish, except the first. From this, we determine the single condition $\lambda_{2} \lambda_{3}=-\nu_{2} \nu_{3}$, which can be satisfied by tuning the external strain magnitude. Under this condition, Dirac points, if present, will be located in the mirror plane on the line parametrized by $k_{3}=\nu_{2} k_{2} / \lambda_{3}$ and $k_{1}=0$.

To focus only on the essential mechanism leading to the existence of Dirac points, we will neglect all terms in $H_{1}$ that do not enter the conditions above explicitly; i.e., we set $g=\lambda_{1}=0, f_{1}=f_{2}=f$, and $\nu_{1}=\nu_{2}=\nu$. We emphasize that this is done merely to simplify our analytical considerations. The results presented below can, however, 


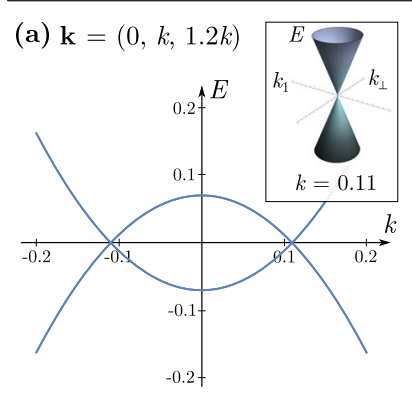

(b)

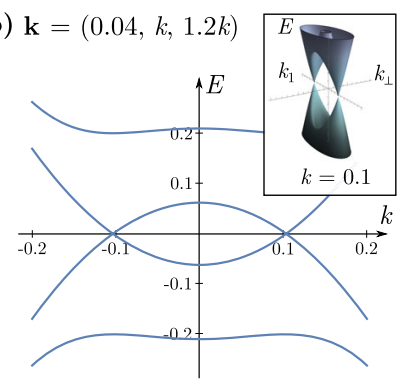

FIG. 2. Spectra of SnTe models with $\lambda_{2}=-1.14 \mathrm{eV}$ and $\lambda_{3}=$ $2.0 \mathrm{eV}$ along a line through the local momentum-space coordinate system centered at $L^{\prime}$. (a) With inversion symmetry: The two bands are each twofold degenerate and cross at two isolated Dirac points. (b) Without inversion symmetry and $\alpha=0.1 \mathrm{eV}$ : The spectrum features four separate Weyl points. The insets show spectra along a perpendicular plane in $\mathbf{k}$ space going through one of the band crossings in the associated main panel. Energies $E$ are given in $\mathrm{eV}$. Momenta $\mathbf{k}$ are displayed in units of $\pi / a$ with the lattice constant $a$.

be generalized also to the full model. With this and the constraints given above, the spectrum along the line $\mathbf{k}=\left(0, k, \nu k / \lambda_{3}\right)$ becomes

$$
E_{ \pm}(k)=\sqrt{\left[\left(\frac{c \nu^{2}}{\lambda_{3}^{2}}+f\right) k^{2}+m\right]^{2}} .
$$

This has the desired form and we infer that Dirac points exist if $m$ and the term before $k^{2}$ have opposite signs. This can be realized by tuning the band mass $m$, e.g., by alloying or pressure [56]. The Dirac points are located at $\pm \mathbf{k}_{0}$ with $\left(k_{0,1}, k_{0,2}, k_{0,3}\right)=\left(0, \sqrt{-m \lambda_{3} /\left(c \nu^{2}+f \lambda_{3}^{2}\right)}, \nu / \lambda_{3} k_{0,2}\right)$. Moreover, an expansion of $\tilde{H}$ around the Dirac points to leading order in $\mathbf{k}$ shows that the effective Hamiltonian is indeed of Dirac form (see Supplemental Material [55]) with, in general, anisotropic dispersion.

We now apply our general results to a specific system by means of numerical calculations. To obtain realistic values for the $\mathbf{k} \cdot \mathbf{p}$ parameters of our model, we fit the parameters of the Hamiltonian $H_{0}$ in Eq. (1) to density functional theory data of SnTe presented in Ref. [51]. From that, we determine the following values (in $\mathrm{eV}$ ): $m=-0.07$, $\nu=2.4, \nu_{3}=0.95, c=0.9$, and $f=4.5$. Next, we introduce a rhombohedral distortion in our SnTe model by tuning $\lambda_{2}$ and $\lambda_{3}$ away from zero until the Dirac-point condition $\lambda_{2}=-\nu \nu_{3} / \lambda_{3}$ is established. The resulting spectrum along a cut through the local coordinate system is shown in Fig. 2(a). We find two Dirac points in agreement with the analytical prediction.

Weyl points and nodal lines.-Bulk Dirac points are, in general, unstable features and can be gapped out by small perturbations. Nonetheless, it is well known that a bulk Dirac point can be split into a pair of stable Weyl points of opposite charge by breaking inversion symmetry.
Furthermore, if mirror symmetry is still present, a Dirac point can also evolve into a nodal line protected by this symmetry [57]. These conditions are naturally realized in the ferroelectric phase of SnTe and GeTe: Below a critical temperature [58] $T_{c}=98 \mathrm{~K}\left(T_{c}=670 \mathrm{~K}\right), \operatorname{SnTe}(\mathrm{GeTe})$ undergoes a structural transition from a rocksalt structure with space group $F m \overline{3} m$ to a rhombohedral lattice with space group $R 3 m[47,48]$. This transition occurs via a twostep symmetry reduction [47-49]. First, an elastic strain along the cube diagonal introduces a rhombohedral distortion and breaks the $C_{4}$ symmetry. This is identical to the symmetry-reduction process discussed above. Second, a relative displacement of the $\mathrm{Sn}(\mathrm{Ge})$ and Te sublattices breaks spatial inversion symmetry, a necessary condition for the semimetallic phases considered in this work, while preserving mirror and $C_{3}$ symmetries. The distorted lattice is illustrated in Fig. 1(a).

To incorporate the second step of the symmetry-reduction procedure into our model, we note that inversionsymmetry breaking reduces the symmetry group of the $L^{\prime}$ points further from $C_{2 h}$ to $C_{s}$; i.e., only the mirror plane remains. In total, there are ten additional symmetry-allowed terms (see Supplemental Material [55]). Here, we restrict our consideration to the following terms:

$$
\begin{gathered}
H_{\alpha}(\mathbf{k})=\alpha \sigma_{x}, \\
H_{\beta}(\mathbf{k})=\beta\left(k_{1} s_{2}-k_{2} s_{1}\right),
\end{gathered}
$$

because each of them gives rise to one of the nodal features described above in a straightforward fashion. We note, however, that also the other inversion-symmetry-breaking terms give rise to the same features.

We next show that the term $H_{\alpha}$ in Eq. (5) splits the Dirac point into two stable Weyl points. For that, we expand the Hamiltonian $\tilde{H}+H_{\alpha}$ around the Dirac point at $\mathbf{k}_{0}$ up to leading order in momentum. The effective Hamiltonian (see Supplemental Material [55]) has the following spectrum:

$$
\begin{aligned}
E^{2}= & \left(2 f k_{0,2} \kappa_{2}+2 c k_{0,3} \kappa_{3}\right)^{2}+\frac{\nu_{3}^{2}}{\lambda_{3}^{2}}\left(\lambda_{3} \kappa_{3}-\nu \kappa_{2}\right)^{2} \\
& +\left[\alpha \pm \sqrt{\left(\lambda_{3} \kappa_{3}-\nu \kappa_{2}\right)^{2}+\left(\nu \kappa_{1}\right)^{2}}\right]^{2} .
\end{aligned}
$$

Since we are again looking for zero-energy states, all terms in parentheses in the equation above must simultaneously vanish. We already know that this is the case for $\alpha=0$. Keeping all parameters fixed except $\alpha$, this implies that zero-energy states must satisfy $\kappa_{2}=\kappa_{3}=0$ even for nonzero $\alpha$. Finally, we obtain zero-energy solutions of Eq. (7) for

$$
\mathbf{k}_{W}=\left( \pm \alpha / \nu, k_{0,2}, k_{0,3}\right) .
$$

The solutions are distinct for $\alpha \neq 0$, and each of them is twofold degenerate. Furthermore, the Weyl points are 
mapped onto each other by reflection about the mirror plane. Since reflection flips the topological charge of a Weyl node [57], we further infer that their topological charge must be opposite. These general findings are confirmed by numerical results as we show in Fig. 2(b): The two Dirac points split into four twofold degenerate states, two on each side of the mirror plane. Furthermore, we calculate the topological charge of each nodal point numerically [59] and obtain nontrivial values of \pm 1 .

We emphasize that, due to their topological charge, the Weyl nodes are robust features of the system and, thus, must appear in an extended region in the parameter space. This implies that we can now explicitly violate the condition $\lambda_{2} \lambda_{3}=-\nu_{2} \nu_{3}$, which led to the existence of Dirac points, or switch on other parameters without gapping out the Weyl nodes (see Supplemental Material [55]). Hence, the Weyl nodes are not subject to parameter fine-tuning, which is in stark contrast to the parent Dirac points.

Because of the presence of mirror symmetry, the decay of a Dirac point into two Weyl points is not the only possible process. In fact, a Dirac point can also evolve into a topologically protected nodal loop located in the mirror plane [57]. We now show that this is exactly what happens using $H_{\beta}$ from Eq. (6) to break inversion symmetry.

As before, we first expand the Hamiltonian $\tilde{H}+H_{\beta}$ around the Dirac point at $\mathbf{k}_{0}$ (see Supplemental Material [55] for the resulting effective Hamiltonian). Let us look at this Hamiltonian along the $k_{1}$ direction perpendicular to the mirror plane. The spectrum along this line is

$$
E\left(\kappa_{1}, 0,0\right)= \pm \sqrt{\left(\beta k_{0,2}\right)^{2}+\kappa_{1}^{2}(\beta \pm \nu)^{2}},
$$

which is always nonzero for $\kappa_{1} \neq 0$, even for infinitesimally small values of $\beta$. Zero-energy states are therefore expected to be located in the mirror plane at $k_{1}=\kappa_{1}=0$.

To obtain the energies in the mirror plane, we look at the spectrum along arbitrary lines through the Dirac point. We parametrize these lines by their slope $\eta$; i.e., we look at lines of the form $(0, \kappa, \eta \kappa)$. We obtain

$$
E_{\eta}(\kappa)= \pm\left(\kappa+k_{0,2}\right) \pm \frac{\sqrt{A_{\eta}}}{\lambda_{3}} \kappa,
$$

where $A_{\eta}=4 \lambda_{3}^{2}\left(f k_{0,2}+c k_{0,3} \eta\right)^{2}+\left(\nu-\lambda_{3} \eta\right)^{2}\left(\lambda_{3}^{2}+\nu_{3}^{2}\right)$. From this, we determine the location of zero-energy states

$$
\mathbf{k}_{N, \eta}=\frac{-\lambda_{3} \beta k_{0,2}}{\lambda_{3} \beta \pm \sqrt{A_{\eta}}}(0,1, \eta) .
$$

Each state is twofold degenerate similar to the Weyl-point solutions above. However, the structure of the solutions is qualitatively different as we show in Supplemental Material [55]: The set of zero-energy states forms a closed line. (a)

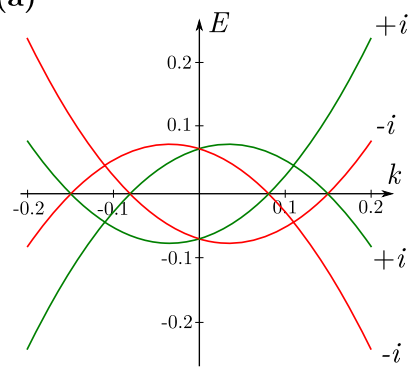

(b)

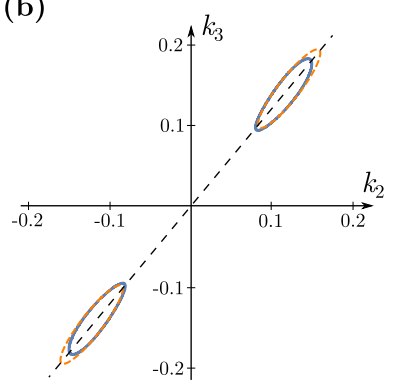

FIG. 3. Spectrum of the SnTe model with $\lambda_{2}=-1.14 \mathrm{eV}$, $\lambda_{3}=2.0 \mathrm{eV}$, and $\beta=0.4 \mathrm{eV}$ (broken inversion symmetry) in the local momentum-space coordinate system centered at $L^{\prime}$. (a) Dispersion along a line parametrized by $\mathbf{k}=(0, k, 1.2 k)$ : There are four twofold degenerate band crossings at zero energy. The mirror eigenvalues of the bands have been indicated in green $(+i)$ and red $(-i)$. (b) $E=0$ Fermi surface (blue lines) in the mirror plane at $k_{1}=0$. The spectrum features two nodal loops. For comparison, the approximate analytical solutions for the nodal lines have been indicated (dashed orange lines). The black dashed line represents the momentum-space cut shown in (a). Energies $E$ are given in $\mathrm{eV}$. Momenta $\mathbf{k}$ are displayed in units of $\pi / a$.

We again check our analytical findings against numerical results for our SnTe model. The spectrum along a cut through the BZ is shown in Fig. 3 along with the $E=0$ Fermi surface in the mirror plane $k_{1}=0$. In accordance with our analytical study, the Dirac points evolve into elliptical twofold degenerate lines located in the mirror plane.

The nodal lines are topologically protected by mirror symmetry $[43,57]$. The mirror plane at $k_{1}=0$, accommodating the nodal lines, is invariant under reflection. Therefore, the mirror operator $M$ and the system Hamiltonian $H(\mathbf{k})$ commute for all momenta in this plane and all states can be assigned a well-defined reflection eigenvalue $\xi= \pm i$. This is illustrated with different colors in Fig. 3(a). We observe that the reflection eigenvalues of occupied states inside the nodal ellipses are different from the ones outside the ellipses; namely, $\xi_{\text {in }}=\{\mp i, \mp i\}$ and $\xi_{\text {out }}=\{\mp i, \pm i\}$ (for $k \gtrless 0$ ). These values cannot change unless the bulk energy gap closes locally, which happens along the nodal lines. Therefore, the nodal lines are protected. The corresponding topological invariant is computed from the difference of occupied states with mirror eigenvalue $-i$ inside and outside the ellipses [43]. We obtain $\nu_{\text {line }}= \pm 1$ for $k \gtrless 0$.

Conclusions. - We have shown that systems in the SnTe material class are a new potential platform for Weyl and nodal line semimetals. The key ingredients are a rhombohedral distortion, induced by an elastic strain along the [111] direction, followed by inversion-symmetry breaking. By deriving and analyzing a minimal $\mathbf{k} \cdot \mathbf{p}$ model, we have demonstrated how this two-step symmetry-reduction process leads to the appearance of topologically stable Weyl nodes. Complementary, we find that the mirror symmetry 
in group-IV tellurides also gives rise to a semimetallic phase featuring topologically protected nodal lines, a class of systems for which only a few candidate materials have been put forward.

There are various, feasible ways to realize our proposal experimentally. First, the conditions for the symmetry reduction are naturally provided in the low-temperature ferroelectric phase of SnTe and GeTe, which could therefore represent novel Weyl ferroelectric semimetals [60,61]. Moreover, additional external strain, pressure, or alloying $[48,49,62-65]$ could be employed to tune the parameters of the systems. In fact, a recent experimental report supports the existence of semimetallic phases in $\mathrm{Pb}$-alloyed $\mathrm{SnTe}$ under pressure [66]. Another route is the use of substrates with different lattice structures. This could induce an inhomogeneous strain close to the substrate interface mimicking a ferroelectric distortion.

Finally, our proposal could also be applied to group-V semimetals such as Bi and Sb [67]. These materials are in the rhombohedral space group $R \overline{3} m$ with an inversion center and have a similar band structure as group-IV tellurides [68]. To realize Weyl nodes or nodal lines, one could therefore use thin films where inversion symmetry can be broken by either using substrates, as explained above, or externally applying a perpendicular electric field.

We thank D. Varjas and T. Rosdahl for helpful discussions regarding the symmetric-Hamiltonian generator algorithm. C. O. acknowledges support from a VIDI grant (Project No. 680-47-543) financed by the Netherlands Organization for Scientific Research (NWO). A. L. acknowledges support from the Netherlands Organisation for Scientific Research (NWO/OCW), as part of the Frontiers of Nanoscience program.

[1] Z. Wang, Y. Sun, X.-Q. Chen, C. Franchini, G. Xu, H. Weng, X. Dai, and Z. Fang, Phys. Rev. B 85, 195320 (2012).

[2] Z. K. Liu, B. Zhou, Y. Zhang, Z. J. Wang, H. M. Weng, D. Prabhakaran, S.-K. Mo, Z.X. Shen, Z. Fang, X. Dai, Z. Hussain, and Y. L. Chen, Science 343, 864 (2014).

[3] S.-M. Huang, S.-Y. Xu, I. Belopolski, C.-C. Lee, G. Chang, B. Wang, N. Alidoust, G. Bian, M. Neupane, C. Zhang, S. Jia, A. Bansil, H. Lin, and M. Z. Hasan, Nat. Commun. 6, 7373 (2015).

[4] H. Weng, C. Fang, Z. Fang, B. A. Bernevig, and X. Dai, Phys. Rev. X 5, 011029 (2015).

[5] B. Q. Lv, H. M. Weng, B. B. Fu, X. P. Wang, H. Miao, J. Ma, P. Richard, X. C. Huang, L. X. Zhao, G. F. Chen, Z. Fang, X. Dai, T. Qian, and H. Ding, Phys. Rev. X 5, 031013 (2015)

[6] B. Q. Lv, N. Xu, H. M. Weng, J. Z. Ma, P. Richard, X. C. Huang, L. X. Zhao, G. F. Chen, C. E. Matt, F. Bisti, V. N. Strocov, J. Mesot, Z. Fang, X. Dai, T. Qian, M. Shi, and H. Ding, Nat. Phys. 11, 724 (2015).

[7] S.-Y. Xu et al., Science 349, 613 (2015).
[8] S.-Y. Xu et al., Nat. Phys. 11, 748 (2015).

[9] O. Vafek and A. Vishwanath, Annu. Rev. Condens. Matter Phys. 5, 83 (2014).

[10] E. Witten, Riv. Nuovo Cimento 39, 313 (2016).

[11] A. A. Burkov, Nat. Mater. 15, 1145 (2016).

[12] B. Yan and C. Felser, Annu. Rev. Condens. Matter Phys. 8, 337 (2017).

[13] N. P. Armitage, E. J. Mele, and A. Vishwanath, Rev. Mod. Phys. 90, 015001 (2018).

[14] A. A. Burkov and L. Balents, Phys. Rev. Lett. 107, 127205 (2011).

[15] C.-K. Chiu and A. P. Schnyder, Phys. Rev. B 90, 205136 (2014).

[16] A. A. Burkov, J. Phys. Condens. Matter 27, 113201 (2015).

[17] A. A. Soluyanov, D. Gresch, Z. Wang, Q. Wu, M. Troyer, X. Dai, and B. A. Bernevig, Nature (London) 527, 495 (2015).

[18] B. Bradlyn, J. Cano, Z. Wang, M. G. Vergniory, C. Felser, R. J. Cava, and B.A. Bernevig, Science 353, aaf5037 (2016).

[19] A. A. Burkov, Phys. Rev. B 96, 041110(R) (2017).

[20] J. Gooth, A. C. Niemann, T. Meng, A. G. Grushin, K. Landsteiner, B. Gotsmann, F. Menges, M. Schmidt, C. Shekhar, V. Süß, R. Hühne, B. Rellinghaus, C. Felser, B. Yan, and K. Nielsch, Nature (London) 547, 324 (2017).

[21] F. de Juan, A. G. Grushin, T. Morimoto, and J. E. Moore, Nat. Commun. 8, 15995 (2017).

[22] E. V. Gorbar, V. A. Miransky, I. A. Shovkovy, and P. O. Sukhachov, Low Temp. Phys. 44, 487 (2018).

[23] G. B. Osterhoudt, L. K. Diebel, M. J. Gray, X. Yang, J. Stanco, X. Huang, B. Shen, N. Ni, P. J. W. Moll, Y. Ran, and K. S. Burch, Nat. Mater. 18, 471 (2019).

[24] A. A. Burkov, Phys. Rev. B 97, 165104 (2018).

[25] X. Wan, A. M. Turner, A. Vishwanath, and S. Y. Savrasov, Phys. Rev. B 83, 205101 (2011).

[26] A. Lau, K. Koepernik, J. van den Brink, and C. Ortix, Phys. Rev. Lett. 119, 076801 (2017).

[27] S. M. Young, S. Zaheer, J. C. Y. Teo, C. L. Kane, E. J. Mele, and A. M. Rappe, Phys. Rev. Lett. 108, 140405 (2012).

[28] S. Murakami, New J. Phys. 9, 356 (2007).

[29] G. B. Halász and L. Balents, Phys. Rev. B 85, 035103 (2012).

[30] P. Hosur, Phys. Rev. B 86, 195102 (2012).

[31] G. Xu, H. Weng, Z. Wang, X. Dai, and Z. Fang, Phys. Rev. Lett. 107, 186806 (2011).

[32] D. Bulmash, C.-X. Liu, and X.-L. Qi, Phys. Rev. B 89, 081106(R) (2014).

[33] J. Ruan, S.-K. Jian, H. Yao, H. Zhang, S.-C. Zhang, and D. Xing, Nat. Commun. 7, 11136 (2016).

[34] A. Lau and C. Ortix, Phys. Rev. B 96, 081411(R) (2017).

[35] N. Xu, H. M. Weng, B. Q. Lv, C. E. Matt, J. Park, F. Bisti, V. N. Strocov, D. Gawryluk, E. Pomjakushina, K. Conder, N. C. Plumb, M. Radovic, G. Autès, O. V. Yazyev, Z. Fang, X. Dai, T. Qian, J. Mesot, H. Ding, and M. Shi, Nat. Commun. 7, 11006 (2016).

[36] K. Deng, G. Wan, P. Deng, K. Zhang, S. Ding, E. Wang, M. Yan, H. Huang, H. Zhang, Z. Xu, J. Denlinger, A. Fedorov, H. Yang, W. Duan, H. Yao, Y. Wu, S. Fan, H. Zhang, X. Chen, and S. Zhou, Nat. Phys. 12, 1105 (2016).

[37] C. Wang et al., Phys. Rev. B 94, 241119(R) (2016). 
[38] K. Koepernik, D. Kasinathan, D. V. Efremov, S. Khim, S. Borisenko, B. Büchner, and J. van den Brink, Phys. Rev. B 93, 201101(R) (2016).

[39] For a more detailed and exhaustive overview of the field, we refer to the excellent review article in Ref. [13].

[40] A. A. Burkov, M. D. Hook, and L. Balents, Phys. Rev. B 84, 235126 (2011).

[41] C. Fang, Y. Chen, H.-Y. Kee, and L. Fu, Phys. Rev. B 92, 081201 (2015).

[42] H. Huang, J. Liu, D. Vanderbilt, and W. Duan, Phys. Rev. B 93, 201114(R) (2016).

[43] C. Fang, H. Weng, X. Dai, and Z. Fang, Chin. Phys. B 25, 117106 (2016).

[44] Q. Xu, R. Yu, Z. Fang, X. Dai, and H. Weng, Phys. Rev. B 95, 045136 (2017).

[45] G. Bian, T.-R. Chang, H. Zheng, S. Velury, S.-Y. Xu, T. Neupert, C.-K. Chiu, S.-M. Huang, D. S. Sanchez, I. Belopolski, N. Alidoust, P.-J. Chen, G. Chang, A. Bansil, H.-T. Jeng, H. Lin, and M. Z. Hasan, Phys. Rev. B 93, 121113(R) (2016).

[46] Y.-H. Chan, C.-K. Chiu, M. Y. Chou, and A. P. Schnyder, Phys. Rev. B 93, 205132 (2016).

[47] K. M. Rabe and J. D. Joannopoulos, Phys. Rev. B 32, 2302 (1985).

[48] E. K. H. Salje, D. J. Safarik, K. A. Modic, J. E. Gubernatis, J. C. Cooley, R. D. Taylor, B. Mihaila, A. Saxena, T. Lookman, J. L. Smith, R. A. Fisher, M. Pasternak, C. P. Opeil, T. Siegrist, P. B. Littlewood, and J. C. Lashley, Phys. Rev. B 82, 184112 (2010).

[49] R. M. Murphy, E. D. Murray, S. Fahy, and I. Savić, Phys. Rev. B 95, 144302 (2017).

[50] L. G. Ferreira, Phys. Rev. 137, A1601 (1965).

[51] T. H. Hsieh, H. Lin, J. Liu, W. Duan, A. Bansi, and L. Fu, Nat. Commun. 3, 982 (2012).

[52] D. L. Mitchell and R. F. Wallis, Phys. Rev. 151, 581 (1966).
[53] D. Varjas, T. O. Rosdahl, and A. R. Akhmerov, New J. Phys. 20, 093026 (2018).

[54] The Python package Qsymm is available at https://gitlab .kwant-project.org/qt/qsymm.

[55] See Supplemental Material at http://link.aps.org/ supplemental/10.1103/PhysRevLett.122.186801 for details of the symmetry analysis, for expansions of the Hamiltonian close the Dirac points, and for a more detailed analysis of the nodal line phase.

[56] P. Barone, T. Rauch, D. Di Sante, J. Henk, I. Mertig, and S. Picozzi, Phys. Rev. B 88, 045207 (2013).

[57] X.-Q. Sun, T. Bzdušek, and S.-C. Zhang, Phys. Rev. Lett. 121, 106402 (2018).

[58] Z.-Y. Ye, H.-X. Deng, H.-Z. Wu, S.-S. Li, S.-H. Wei, and J.-W. Luo, npj Comput. Mater. 1, 15001 (2015).

[59] T. Fukui, Y. Hatsugai, and H. Suzuki, J. Phys. Soc. Jpn. 74, 1674 (2005).

[60] D. Di Sante, P. Barone, A. Stroppa, K. F. Garrity, D. Vanderbilt, and S. Picozzi, Phys. Rev. Lett. 117, 076401 (2016).

[61] R. Li, Y. Xu, J. He, S. Ullah, J. Li, J.-M. Liu, D. Li, C. Franchini, H. Weng, and X.-Q. Chen, arXiv:1610.07142.

[62] J. O. Dimmock, I. Melngailis, and A. J. Strauss, Phys. Rev. Lett. 16, 1193 (1966).

[63] A. J. Strauss, Phys. Rev. 157, 608 (1967).

[64] S. Jin, C. Cai, G. Bi, B. Zhang, H. Wu, and Y. Zhang, Phys. Rev. B 87, 235315 (2013).

[65] A. Lusakowski, P. Boguslawski, and T. Story, Phys. Rev. B 98, 125203 (2018).

[66] T. Liang, S. Kushwaha, J. Kim, Q. Gibson, J. Lin, N. Kioussis, R. J. Cava, and N. P. Ong, Sci. Adv. 3, e1602510 (2017).

[67] Y. Liu and R. E. Allen, Phys. Rev. B 52, 1566 (1995).

[68] S. A. Gordyunin and L. P. Gor'kov, JETP Lett. 20, 307 (1974). 